Konferenz der unabhängigen Datenschutzbehörden des Bundes und der Länder

\section{Entschließung vom 20. April 2016}

\section{Klagerecht für Datenschutzbehörden - EU-Kommissionentscheidungen müssen gerichtlich überprüfbar sein}

Die Konferenz der unabhängigen Datenschutzbehörden des Bundes und der Länder (Datenschutzkonferenz) fordert den Gesetzgeber auf, umgehend ein eigenständiges Klagerecht für die unabhängigen Datenschutzbehörden vorzusehen. Wenn die unabhängigen Datenschutzbehörden der Auffassung sein sollten, dass eine Entscheidung der EUKommission rechtswidrig ist, wären sie gleichwohl an diese gebunden. Sie müssten daher ggf. gegen den rechtsstaatlichen Grundsatz der Gesetzmäßigkeit der Verwaltung verstoßen. Um dies zu verhindern, sind die prozessualen Voraussetzungen dafür zu schaffen, dass die Datenschutzbehörden selbst bestehende Zweifel an der Rechtmäßigkeit einer Kommissionsentscheidung gerichtlich klären lassen können.

Anlass für die obige Aufforderung der Datenschutzkonferenz ist die zwischenzeitliche Vorlage einer Reihe von Dokumenten unterschiedlicher Repräsentanten der USAdministration durch die EU-Kommission am 29. Februar 2016, die für Unternehmen und Behörden Zusagen für den Umgang mit aus der EU übermittelten personenbezogenen Daten enthalten. Im Rahmen eines so genannten EU-US Privacy Shield sollen diese Dokumente Grundlage für eine künftige EU-Kommissionsentscheidung zur Angemessenheit des Datenschutzniveaus in den USA sein und damit als Nachfolgeregelung für die Safe Harbor-Entscheidung dienen. Letztere wurde bekanntlich am 6. Oktober 2015 durch den Europäischen Gerichtshof aufgehoben.

Gegen den „EU-US Privacy Shield“ bestehen jedoch nach Auffassung der Artikel29-Datenschutzgruppe, dem Zusammenschluss der Datenschutzbehörden der EU-Mitgliedstaaten und des Europäischen Datenschutzbeauftragten, erhebliche Bedenken. Die Artikel-29-Dateschutzgruppe hat zum „EU-US Privacy Shield“ zuletzt am 13. April 2016 detailliert Stellung genommen. ${ }^{1}$ Die Datenschutzkonferenz teilt diese umfassende Analyse und unterstützt die darin enthaltene Forderung an die EU-Kommission, vor einer Beschlussfassung substantielle Nachbesserungen vorzunehmen. Die Datenschutzkonferenz ist der Auffassung, dass auch der „EU-US Privacy Shield“ in seiner derzeitigen Form nicht ausreichend ist, das für die Übermittlung personenbezogener Daten in die USA erforderliche ,angemessene Datenschutzniveau“ in den USA zu gewährleisten.

Der EuGH stellt in seiner o. g. Entscheidung zur Ungültigkeit von Safe Harbor ausdrücklich klar, dass nach Maßgabe der Datenschutz-Richtlinie der nationale Gesetzgeber für die Datenschutzbehörden Rechtsbehelfe vorzusehen hat, die ihnen bei rechtlichen Zweifeln über eine Angemessenheitsentscheidung die Anrufung nationaler Gerichte ermöglichen, so dass diese den EuGH um eine Entscheidung über die Vereinbarkeit mit den europäischen Grundrechten ersuchen können.

Die Datenschutzkonferenz begrüßt und unterstützt daher ausdrücklich die Bundesratsinitiative der Freien und Hansestadt Hamburg zur zeitnahen Einräumung eines Klagerechts für die Datenschutzaufsichtsbehörden von Bund und Ländern (BR-Drs. 171/16), in der nochmals deutlich gemacht wird, „dass das vom Europäischen Gerichtshof (EuGH in seinem Urteil vom 6.10.2015 (Rechtssache C-362/14) statuierte Klagerecht für Datenschutzaufsichtsbehörden für die Gewährleistung einer effektiven Datenschutzkontrolle von besonderer Bedeutung ist"

\section{Springer Vieweg}
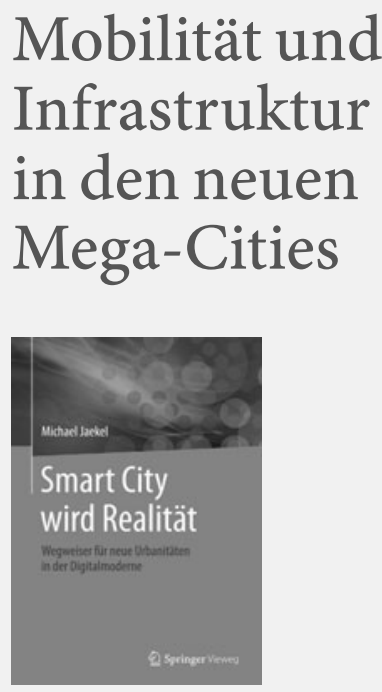

Michael Jaekel

Smart City wird Realität

1. Aufl. 2015. XVI, 312 S.

$108 \mathrm{Abb}$. Brosch.

$€(D) 49,99|€(A) 51,39|^{*} \operatorname{sFr} 53,00$

ISBN 978-3-658-04454-1 (Print)

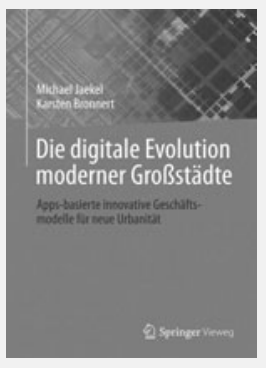

Michael Jaekel; Karsten Bronnert Die digitale Evolution moderner Großstädte

2013. X, 190 S. 51 Abb. Brosch. $€$ (D) $52,99|€(A) 54,47|{ }^{*} \mathrm{sFr} 56,00$ ISBN 978-3-658-00170-4 (Print)

$€(D)$ sind gebundene Ladenpreise in Deutschland und enthalten $7 \%$ MwSt. $€$ (A) sind gebundene Ladenpreise in Österreich und enthalten $10 \%$ MwSt. Die mit * gekennzeichneten Preise sind unverbind liche Preisempfehlungen und enthalten die landesübliche MwSt. Preisänderungen und Irrtümer vorbehalten.

springer-vieweg.de

1 DuD Heft 5 2016, S406 